Felipe d. A. Dias*, Philipp Wiedemann, Marco J. da Silva, Eckhard Schleicher, and Uwe Hampel

\title{
Tuning capacitance wire-mesh sensor gains for measurement of conductive fluids
}

\author{
Optimierung eines kapazitiven Gittersensors für die Messung von leitfähigen Flüssigkeiten
}

DOI 10.1515/teme.2021.0055

\begin{abstract}
In this paper, the front-end circuit of a capacitance wire-mesh sensor (WMS) is analyzed in detail and a new methodology to tune its feedback gains is reported. This allows, for the first time, a capacitance WMS to be able to provide linear measurements of multiphase fluids with electrical conductivity greater than $100 \mu \mathrm{S} / \mathrm{cm}$, which is particularly important for tap water, where the conductivity is typically in between $100 \mathrm{~S} / \mathrm{cm}$ and $500 \mu \mathrm{S} / \mathrm{cm}$. Experimental and numerical results show that the selected gains using the proposed methodology contribute to suppress cross-talk and energy losses, which in turn, reduces considerably the deviation of the conductivity measurement and the estimation of derived flow parameters, such as local and average phase fraction.
\end{abstract}

Keywords: capacitance wire-mesh sensor, multiphase flow, electronic circuit modelling, finite-element method

Zusammenfassung: In diesem Beitrag wird die FrontEnd-Schaltung eines kapazitiven Gittersensors (WMS) im Detail analysiert und eine neue Methodik zur Abstimmung seiner Rückkopplungsverstärkungen beschrieben. Damit kann erstmals ein kapazitives WMS linear Messungen von mehrphasigen Flüssigkeiten mit einer elektrischen Leitfähigkeit von mehr als $100 \mu \mathrm{S} / \mathrm{cm}$ durchführen, was besonders für Leitungswasser wichtig ist, bei dem die Leitfähigkeit typischerweise zwischen $100 \mu \mathrm{S} / \mathrm{cm}$ bis $500 \mu \mathrm{S} / \mathrm{cm}$ liegt. Experimentelle und numerische Ergebnisse zeigen, dass die ausgewählten Verstärkungen mit der vorgeschlagenen Methodik dazu beitragen, Übersprechen und Energieverluste zu unterdrücken, was wiederum

\footnotetext{
*Corresponding author: Felipe d. A. Dias, Helmholtz-Zentrum Dresden-Rossendorf, Institute of Fluid Dynamics, Dresden, Germany, e-mail: f.dias@hzdr.de

Philipp Wiedemann, Eckhard Schleicher, Uwe Hampel, Helmholtz-Zentrum Dresden-Rossendorf, Institute of Fluid Dynamics, Dresden, Germany, e-mail: p.wiedemann@hzdr.de, e.schleicher@hzdr.de, u.hampel@hzdr.de

Marco J. da Silva, Universidade Tecnológica Federal do Paraná, Department of Electrical and Computer Engineering (CPGEI), Curitiba-PR, Brazil, e-mail: mdasilva@utfpr.edu.br
}

die Messabweichungen der Leitfähigkeitsmessung und die Schätzung abgeleiteter Strömungsparameter, wie lokaler und mittlerer Phasenanteil, erheblich reduziert.

Schlüsselwörter: Kapazitiver Gittersensor, Mehrphasenströmung, elektronische Schaltungssimulation, FiniteElemente Methode

\section{Introduction}

Wire-mesh sensor (WMS) is a multiphase flow imaging device with high temporal and spatial resolution. Its operation principle is based on the measurement of at least one electrical property of the fluid, i.e. conductivity [10], permittivity $[1,11]$ or both $[2,5]$. Thus, derived parameters of the flow can be obtained from such electrical properties, e.g. gas/liquid hold up, concentration of an ionic tracer, etc.

In the WMS proposed by da Silva, Schleicher und Hampel [11], the sensor was originally optimized to measure fluids with conductivity lower than $100 \mu \mathrm{S} / \mathrm{cm}$. Since the signal response can be approximated as purely capacitive in this conductivity range, the sensor was introduced to the scientific community as capacitance wire-mesh sensor.

When a standard capacitance WMS is used to measure mixtures with conductivity greater than $100 \mu \mathrm{S} / \mathrm{cm}$, the signal response may become purely conductive. Since the sensor was not designed to operate in this range, the WMS data suffers from non-linearities, e.g. cross-talk and energy losses. Consequently, advanced algorithms (e.g. $[2,3])$ are necessary to relate WMS raw data to derived flow parameters instead of a simple linear relationship as proposed by Prasser, Böttger und Zschau [10] and Silva, Schleicher und Hampel [11].

More recently, Dias, Wiedemann, Da Silva, Schleicher und Hampel [4] has demonstrated that the causes of nonlinearities on capacitance WMS data are mostly related to AC limitations of the external circuits coupled to the sensor, e.g. open-loop gain and bandwidth of the amplifiers. Thus, if ideal amplifiers could be used, the capacitance 


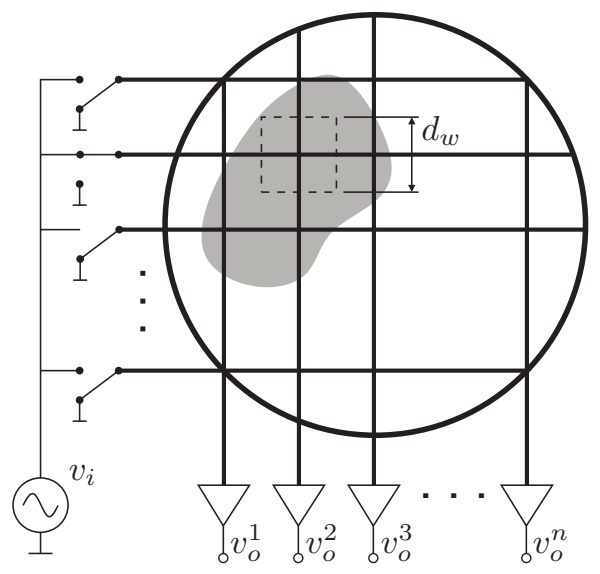

(a) Front view.

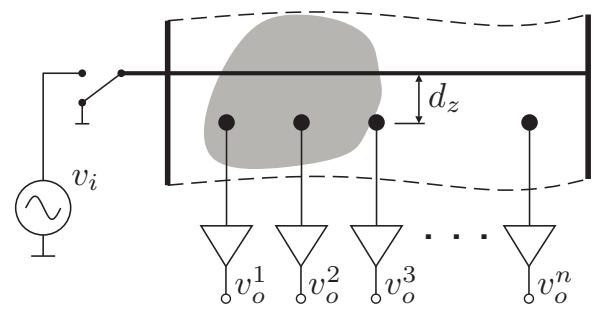

(b) Lateral view.

Fig. 1: Simplified schematic of a standard WMS.

WMS would be able to provide linear readings for fluids with conductivity greater than $100 \mu \mathrm{S} / \mathrm{cm}$.

With that in mind, in this paper we first analyze the front-end circuitry of a WMS taking into account nonideal behavior and secondly propose a method to find a set of feedback gains which makes the non-ideal amplifiers of a WMS closer to ideal. Hence, lower deviations in the phase fraction estimation are achieved.

\section{Wire-mesh sensor}

\subsection{Principle of operation}

Figure 1 shows a schematic of a standard WMS. As can be seen, its electrode wires are stretched over the cross section forming a grid with two planes of orthogonal wires, which are transmitter and receiver wires, respectively, axially separated by a small distance $d_{z}$ (Figure 1b). Each crossing-point represents an individual measuring cell, which is interrogated by an AC-voltage.

The input signal is multiplexed by switches in such a way that only a single transmitter electrode is activated at a time, while the other transmitters are grounded and all receivers are synchronously sampled. At the end of

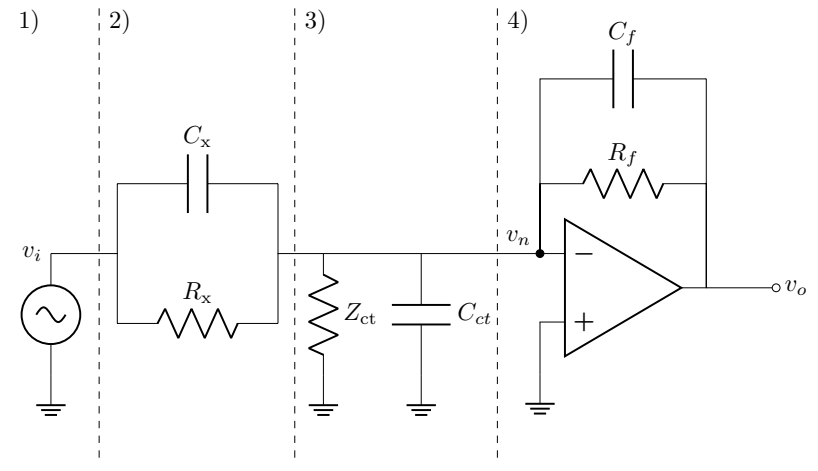

Fig. 2: Equivalent circuit model of a single channel of WMS composed by four parts: 1) excitation signal source; 2) impedance of the fluid in the control volume; 3 ) impedance associated to the neighboring crossing-points relative to the interrogated one / stray capacitance; and 4) inverting amplifier.

a measurement cycle, a 2D matrix containing the signal response of all crossing-points is obtained, which is usually processed offline to derive flow parameters, such as gas void fraction and liquid hold-up. More recently, efforts have been made to create an industrial WMS for robust and real-time measurement $[9,12]$.

\subsection{Idealized model of a crossing-point}

A single channel of a WMS can be approximated by an equivalent electrical circuit [1] (Figure 2). Assuming that the amplifier is ideal, the node $v_{n}$ is perfectly grounded due to the virtual ground. Hence, the impedance $Z_{c t}$ and stray capacitance $C_{c t}$ can be neglected. Note that the load in the receiver wire can be represented as an equivalent impedance $Z_{c t} \| C_{c t}$. However, it has been represented separately to distinguish different sources of stray currents. $Z_{c t}$ represents the impedance associated to the neighboring crossing-points of the activated one and $C_{c t}$ the stray capacitance from cables, PCB layout, etc. The system response can be simplified as

$$
H_{x}^{i d e a l}(j \omega)=\frac{v_{o}(j \omega)}{v_{i}(j \omega)}=-\frac{\left(G_{x}+j \omega C_{x}\right)}{\left(G_{f}+j \omega C_{f}\right)} .
$$

where, $G_{x}$ and $C_{x}$ are the conductance and capacitance of the interrogated fluid. $G_{f}$ and $C_{f}$ are external components connected to the operational amplifier to set the system feedback gain. $v_{i}$ and $v_{o}$ are the input and output signals, $\omega$ is the angular frequency, $H_{x}^{\text {ideal }}$ is the ideal closed loop gain and $j=\sqrt{-1}$. The capacitance and conductance of the fluid are estimated as

$$
C_{x}=\varepsilon_{o} \varepsilon_{x} k_{g},
$$




$$
G_{x}=\sigma_{x} k_{g},
$$

where, $\varepsilon_{x}$ and $\sigma_{x}$ are the relative permittivity and conductivity of the fluid, respectively, $\varepsilon_{o}$ is the vacuum permittivity and $k_{g}$ is a geometry factor of the measuring cell, which is assumed constant for both electrical properties. In this work, $k_{g}$ has been simplified as a rectangular cuboid with volume $d_{w}^{2} \cdot d_{z}^{-1}$, where $d_{z}$ is the axial distance between transmitter and receiver wires and $d_{w}$ is the distance between two parallel wires (see Fig. 1).

\subsection{Non-ideal model of a crossing-point}

According to Dias, Wiedemann, Da Silva, Schleicher und Hampel [4], the non-ideal characteristics of the amplifiers limit the performance of the WMS depending on the flow condition and conductivity of the fluid. Thus, such nonideal characteristics should be considered in order to tune the gains of the sensor properly.

As presented in [7], a non-ideal amplifier can be approximated as

$$
H_{x}^{\text {nonideal }}(j \omega)=\frac{H_{x}^{\text {ideal }}(j \omega)}{1+\frac{1}{a(j \omega) \beta(j \omega)}},
$$

where,

$$
a(j \omega)=\frac{a_{o}}{1+\frac{j \omega}{\omega_{c}}}
$$

is the open-loop gain, and

$$
\beta(j \omega)=\frac{Y_{f}}{Y_{f}+Y_{x}+Y_{c t}+C_{c t}}
$$

is the feedback factor.

The constants $a_{o}$ and $\omega_{c}$ are the static gain and cut-off frequency of the amplifier, respectively and $Y$ represents the admittances in the equivalent circuit shown in Figure 2

Equation (6) is found using the same procedure as presented by Filho, Costa, Cunha, Belfort, Catunda, dos Santos und da Silva [6]. However, in our work, we included the impedance $Z_{c t}$ (or the reciprocal $Y_{c t}$ ) to consider coupling effects of the crossing-points neighboring the activated one.

The magnitude error of $H(j \omega)$ [7] is calculated by

$$
\epsilon_{x}=\left|\frac{1}{1+\frac{1}{a(j \omega) \beta(j \omega)}}\right|-1,
$$

which is finally used as a performance indicator to find suitable feedback gains.

\section{Materials and Methods}

\subsection{Method to tune WMS gains}

The gains of a capacitance wire-mesh sensor are usually tuned experimentally by choosing $R_{f}$ and $C_{f}$ in order to get the highest output signal (without saturation) when the sensor is fully submerged in water. This strategy has worked successfully for deionized water, however, if the conductivity of the mixture is greater than $100 \mu \mathrm{S} / \mathrm{cm}$, the measurement becomes nonlinear [4].

According to Dias, Wiedemann, Da Silva, Schleicher und Hampel [4], such nonlinearities are caused by AC limitations of the transimpedance amplifiers connected to the WMS electrodes. If the gains are not well tuned, the virtual ground of the amplifiers (node $v_{n}$ from Figure 2) differs from zero depending on the phase distribution. Consequently, undesirable currents might flow from the activated channel to the neighboring wires due to $C_{c t}$ and $Z_{c t}$, causing cross-talk and energy losses.

Such problems, however, can be mitigated by selecting feedback gains which minimize the magnitude error calculated by (7). The mappings from Figure 3(a)-(b) show the relationship between the gains $R_{f}, C_{f}$ and the closed-loop error for deionized $(1 \mu \mathrm{S} / \mathrm{cm})$ and tap water $(500 \mu \mathrm{S} / \mathrm{cm})$, respectively. Both mappings were calculated based on the following assumptions:

- Amplifier: $a_{o}=60 \mathrm{~dB}$ and GBW $=230 \mathrm{MHz}$;

- Geometry factor: $k_{g}=d_{w}^{2} \cdot d_{z}^{-1}$ (see Fig. 1);

- Impedance of non-activated channels: $Z_{c t}=Z_{x}$;

- Stray capacitance: $C_{c t}=120 \mathrm{pF}[8]$;

When the sensor is filled with deionized water (Figure 3a), the majority of gain combinations yield deviation lower than $0.1 \%$. In contrast, if the sensor contains tap water, only a few regions provide low error (Figure $3 b$ ).

The hatched red areas indicate signal saturation. In the case of tap water, if the gains are selected aiming the maximum output signal (close to the hatched area), the amplifier error increases considerably. Here, three combination of gains were selected to evaluate the impact of the amplifier error on WMS readings (red stars from Figure $3 b)$.

\subsection{Numerical and experimental evaluation}

The evaluation of the selected gains was made by numerical simulation and by experiments. In the numerical 


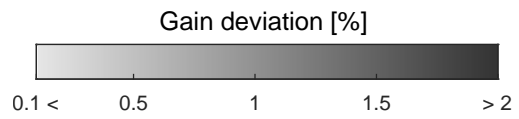

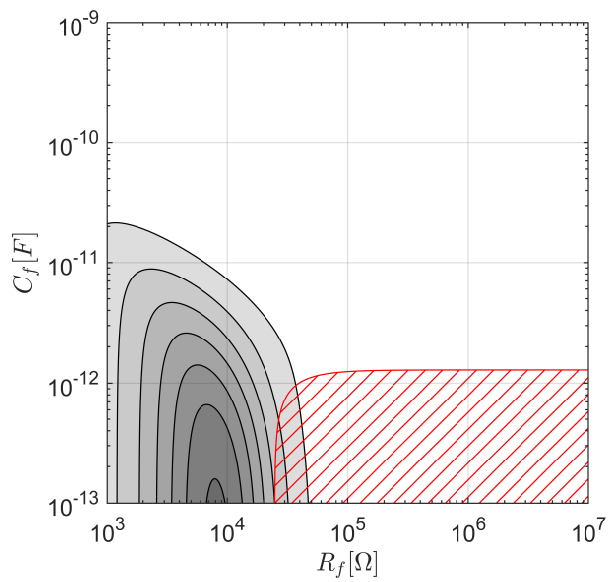

(a) Deionized water

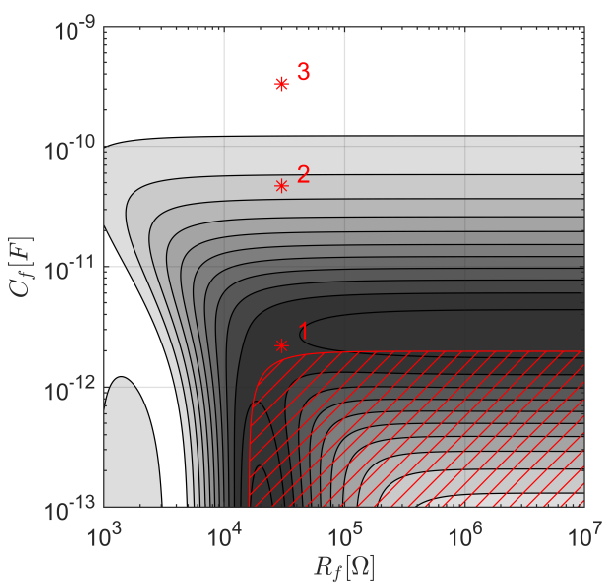

(b) Tap water

Fig. 3: Magnitude error distribution of $H(j \omega)$ calculated by Equation (7). The hatched area represents the gains where the signal response of WMS saturates. The white regions mean deviations smaller than $0.1 \%$ and the red stars are the selected gains used in the evaluation.

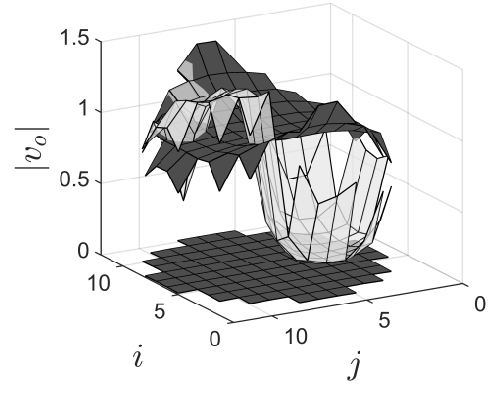

(a) Pattern $1 ; C_{f}=2.2 \mathrm{pF}$

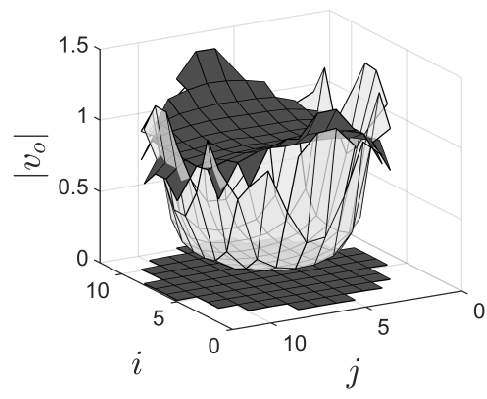

(d) Pattern 2; $C_{f}=2.2 \mathrm{pF}$

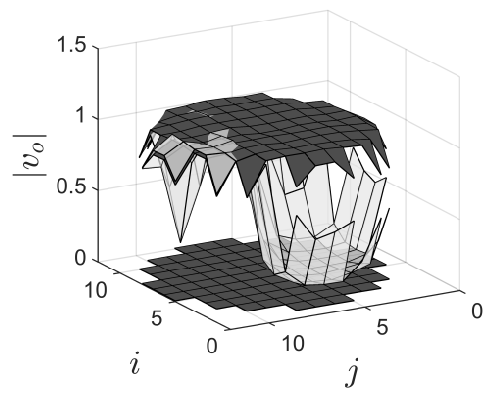

(b) Pattern $1 ; C_{f}=47 \mathrm{pF}$

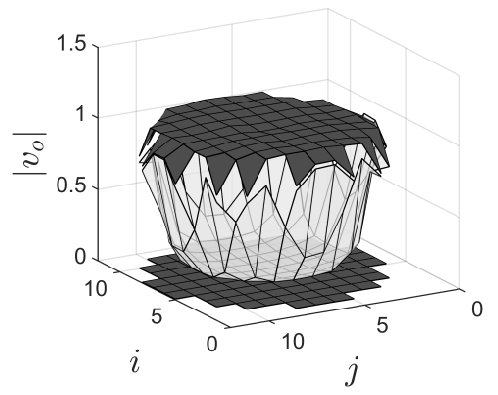

(e) Pattern 2; $C_{f}=47 \mathrm{pF}$

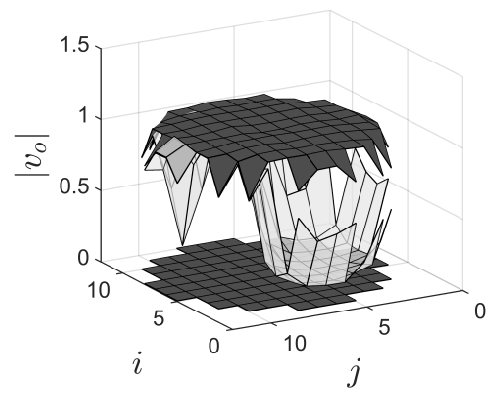

(c) Pattern 1; $C_{f}=330 \mathrm{pF}$

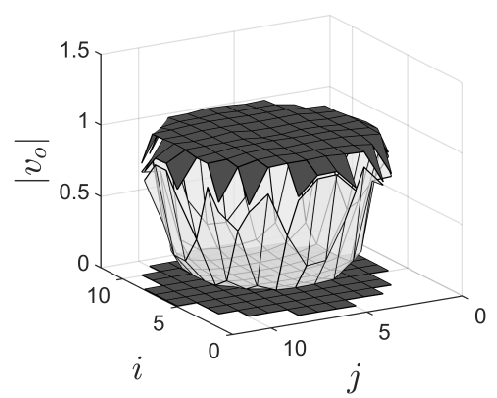

(f) Pattern 2; $C_{f}=330 \mathrm{pF}$

Fig. 4: 3D visualization of synthetic data obtained from the FEM+EC model based on the patterns shown in Figure 5(a). Z-axis is the normalized output voltage $v_{o}$ and the $i$ and $j$ represent the index of the crossing-points (lines and columns, respectively). The dark gray surfaces represent the calibration data and light gray the measurement data. The upper dark surfaces represent $100 \%$ tap water and the lower ones represent $100 \%$ air. (a) and (d) show distortions in the measurement and calibration data caused by deviations in the amplifiers; (b)-(c) and (e)-(f) show linearized data by selecting correct feedback gains. 

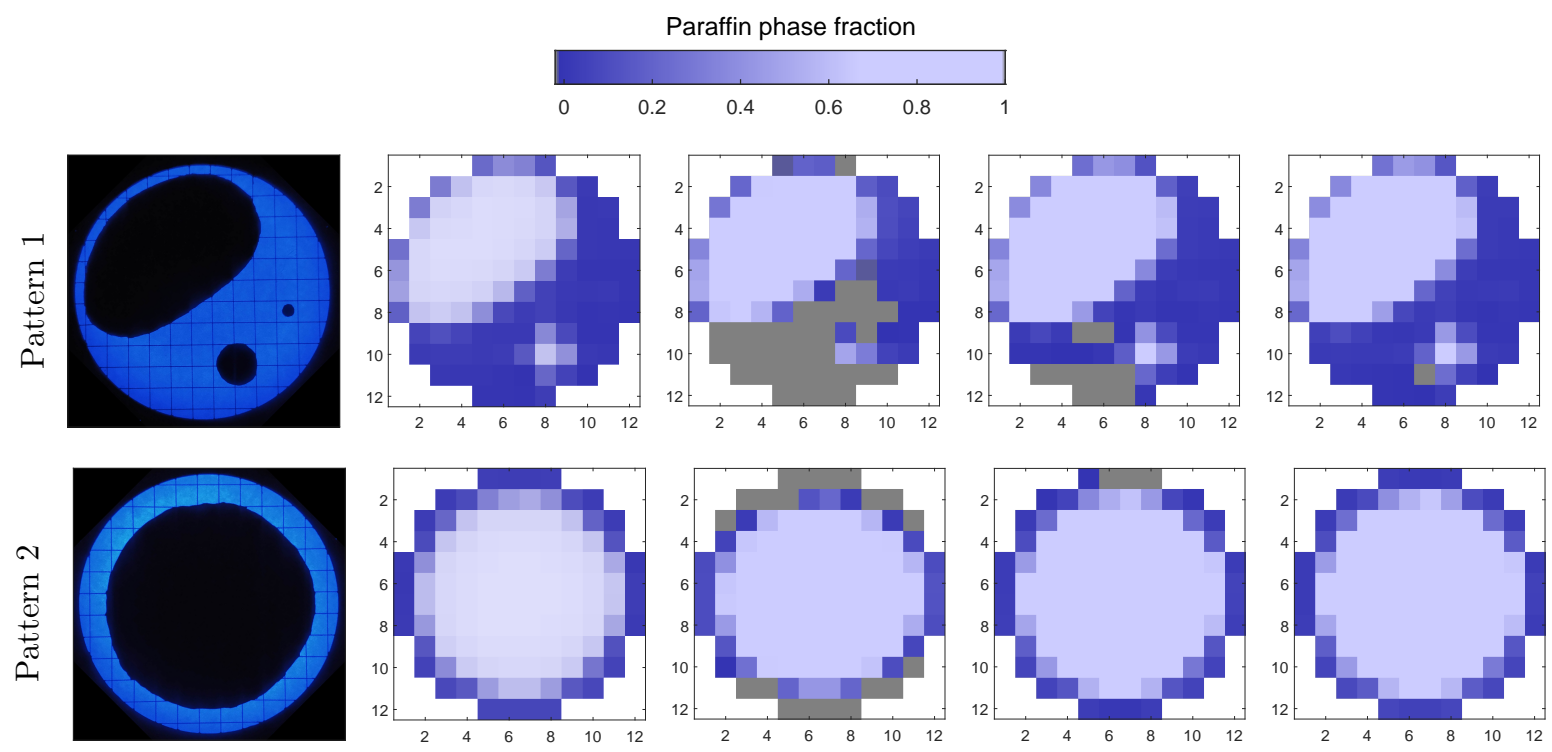

(a) Reference

(b) Basic FEM

\begin{abstract}
(c) $\mathrm{FEM}+\mathrm{EC}$
\end{abstract}
(d) $\mathrm{FEM}+\mathrm{EC}$

(e) $\mathrm{FEM}+\mathrm{EC}$

$\left(C_{f}=2.2 \mathrm{pF}\right)$

$\left(C_{f}=47 \mathrm{pF}\right)$

$\left(C_{f}=330 \mathrm{pF}\right)$

Fig. 5: Synthetic frames obtained by FEM [4]; (a) Reference phase fraction distribution used in the FEM simulation (black patterns in the pipe represent paraffin phase $(\sigma=0 ; \varepsilon=2.5)$; blue is water $(\sigma=420 \mu \mathrm{S} / \mathrm{cm} ; \varepsilon=80)$ and black outside the pipe is not considered in the simulation) (More details in [4]); (b) Phase fraction distribution estimated by the ideal FEM model ; (c)-(e) Phase fraction distribution estimated by the FEM model coupled to external circuits. Gray pixels represent phase fraction saturation: $\alpha<0 \cup \alpha>1$.
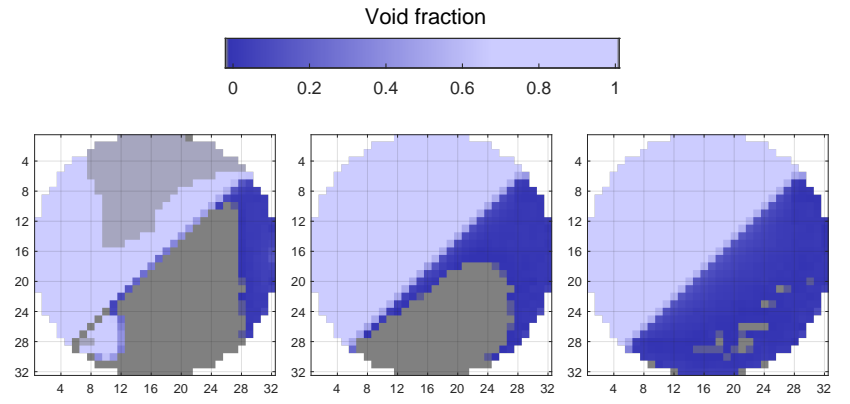

(a) $C_{f}=2.2 \mathrm{pF}$

(b) $C_{f}=47 \mathrm{pF}$

(c) $C_{f}=330 \mathrm{pF}$

Fig. 6: Experimental gas void fraction distribution measured by a $32 \times 32$ WMS containing $50 \%$ water. (a)-(c) Resulting frames for different feedback capacitors $C_{f}$. Gray pixels represents phase fraction saturation: $\alpha<0 \cup \alpha>1$.

simulation, synthetic data from a $12 \times 12$ WMS were generated through two FEM models (the reader is referred to our previous work [4] for details). In the first model, the amplifiers were approximated as macro models and coupled to a 3D sensor geometry, allowing different gains to be tested. This model is referred to as (FEM+EC). In the second model, only the electric field within the sensor was considered. For this reason, it is referred as ideal/basic FEM.

In the experimental evaluation, a $32 \times 32$ WMS was assembled with two acrylic flanges closed on both sides.
However, one of the flanges contained a hole for injecting or removing water. The sensor was connected to the acquisition system commercialized by HZDR INNOVATION $G m b H$. Here, the amplifier gains of the receiver modules were modified according to the selected points shown in Figure 3b.

The acquisition was performed at a frequency of $2.5 \mathrm{kHz}$ for 1 minute each. The raw data were then converted to phase fraction by means of a static calibration, that is, with measurements from the sensor empty and fully filled with tap water $(\sigma \approx 420 \mu \mathrm{S} / \mathrm{cm})$. The estimation of the phase fraction was performed using the parallel mixture model (linear model), as proposed in [11].

\section{Results}

Figure 4 shows 3D visualizations of synthetic data generated by the FEM+EC model. As can be seen, the closed loop gain of the amplifiers is fundamental to obtain linear measurements $0 \leq\left|v_{o}\right| \leq 1$. When the capacitor $C_{f}$ is set to $2.2 \mathrm{pF}$, the output error is large and a voltage appears at node $V_{n}$, as discussed earlier. In addition, as the impedance $Z_{c t}$ is different for each crossing-point, i.e. higher for crossing-points closer to the pipe wall and smaller for crossing-points in the middle, the water cali- 
Table 1: Average phase fraction deviation of synthetic and experimental data estimated without the cut-off method.

\begin{tabular}{rccccc|ccc}
\hline & Basic FEM & \multicolumn{3}{c|}{ FEM+EC } & \multicolumn{3}{c}{ Experiment } \\
$C_{f}$ & - & $\mathbf{2 . 2} \mathbf{p F}$ & $\mathbf{4 7} \mathbf{p F}$ & $\mathbf{3 3 0} \mathbf{~ p F}$ & $\mathbf{2 . 2} \mathbf{p F}$ & $\mathbf{4 7} \mathbf{p F}$ & $\mathbf{3 3 0} \mathbf{~ p F}$ \\
\hline Pattern 1 & $0.5 \%$ & $17.0 \%$ & $3.2 \%$ & $2.2 \%$ & - & - & - \\
Pattern 2 & $0.4 \%$ & $19.6 \%$ & $3.3 \%$ & $2.3 \%$ & - & - & - \\
Stratified & - & - & - & - & $20.3 \%$ & $5.1 \%$ & $2.8 \%$ \\
\hline
\end{tabular}

bration data (upper dark surface in Figure 4(a) and 4(d)) presents a curvature in the points located in the middle of the flow domain caused by energy losses.

As the amplifier error decreases with the increase of $C_{f}$ (Figure 3b), the non-ideal amplifier works closer to an ideal one. Consequently, the effects of the impedance $Z_{c t}$ and the cacpacitance $C_{c t}$ are suppressed and the measurement becomes linear, as shown in Figure 4(b-c) and 4(e-f).

A direct consequence of poorly tuned gains is the negative phase fraction estimation. This problem may also occur due to temperature drifts [13] and nonlinearities of the electric potential [14]. However, Dias, Wiedemann, Da Silva, Schleicher und Hampel [4] have shown that the most critical cause of negative phase fraction is related to the amplifiers. This situation is illustrated in the Figures 5(c)-(d) (gray pixels). Here, the synthetic data was calibrated using the parallel model (linear approximation) without cut-off method [11]. In the frames generated by the basic FEM model (Figure 5(b)), the amplifiers are not considered. For this reason, negative phase fraction is not appreciated in the images. In the FEM+EC model, the gain that provides the lower number of gray pixels is the one with selected gains that provides the least closed-loop error, as expected according to the mapping from Figure $3(\mathrm{~b})$.

In a sensor with a large diameter, the impedance $Z_{c t}$ is much smaller in comparison with small sensors, since the activated crossing points are surrounded by larger volumes of conductive fluid. Figure 6 shows, however, that even in this condition, by increasing the capacitor $C_{f}$ the number of pixels representing negative void fraction can be drastically reduced. This experimental result was obtained from a $32 \times 32$ WMS (8 inch diameter).

Table 1 summarizes the average phase fraction estimation from synthetic (Figure 5) and experimental (Figure 6) data. As can be seen, by using well-tuned gains, the phase fraction deviation was reduced from $20.3 \%$ to $2.8 \%$ in the worst case (experimental measurement with large sensor). These results demonstrate the potential of the WMS proposed in [11] to measure not only capacitive, but also conductive fluids with very good accuracy.

\section{Conclusion}

In this paper, a detailed analysis and new method to tune the feedback gains of capacitance wire-mesh sensor is presented. With this method, a set of feedback gains of the amplifiers of WMS were selected, enabling the sensor to provide linear measurements of conductive fluids such as tap water $(\sigma \approx 420 \mu \mathrm{S} / \mathrm{cm})$ for the first time. Experimental results showed a reduction on the average phase fraction deviation from $20.3 \%$ to $2.8 \%$ due to the interchange of gains.

In near future, the new method combined with FEM will be used to optimize the external circuits of WMS in order to extend its conductivity range.

Funding: This research was funded by the European Social Fund and co-financed by tax funds based on the budget approved by the members of the Saxon State Parliament. Mr. Dias was funded by the European Social Fund and the Free State of Saxony, reference number: $100316833 / 100316834$.

\section{References}

[1] M. J. Da Silva. Impedance sensors for fast multiphase flow measurement and imaging, 2008.

[2] F. D. A. Dias, E. N. dos Santos, M. J. da Silva, E. Schleicher, R. E. M. Morales, B. Hewakandamby und U. Hampel. New algorithm to discriminate phase distribution of gas-oil-water pipe flow with dual-modality wire-mesh sensor. IEEE Access, 8:125163-125178, 2020. 10.1109/ACCESS.2020.3007678.

[3] F. D. A. Dias, D. R. Pipa, R. E. M. Morales und M. J. d. Silva. Wire-mesh sensor super-resolution based on statistical reconstruction. IEEE Transactions on Instrumentation and Measurement, 70:1-12, 2021. 10.1109/TIM.2021.3058362.

[4] F. D. A. Dias, P. Wiedemann, M. J. Da Silva, E. Schleicher und U. Hampel. Combined finite element and electronic circuit model of a wire-mesh sensor. IEEE Access, 9:6630966322, 2021. 10.1109/ACCESS.2021.3076966.

[5] E. N. dos Santos, T. P. Vendruscolo, R. E. M. Morales, E. Schleicher, U. Hampel und M. J. D. Silva. Dual-modality wire-mesh sensor for the visualization of three-phase flows. 
Measurement Science and Technology, 26(10):105302, sep 2015. 10.1088/0957-0233/26/10/105302.

[6] J. B. S. Filho, C. A. M. Costa, G. C. L. Cunha, D. R. Belfort, S. Y. C. Catunda, E. N. dos Santos und M. J. da Silva. Requirements for an integrated conditioning circuit for multiphase flow imaging using impedance wire-mesh sensors. In 2016 IEEE 7th Latin American Symposium on Circuits Systems (LASCAS), S. 291-294, 2016. 10.1109/LASCAS.2016.7451067.

[7] S. Franco. Design with operational amplifiers and analog integrated circuits, Band 1988. McGraw-Hill New York, 2002. ISBN 978-0078028168.

[8] J. C. Gamio, W. Q. Yang und A. L. Stott. Analysis of nonideal characteristics of an ac-based capacitance transducer for tomography. Measurement Science and Technology, 12(8): 1076-1082, jul 2001. 10.1088/0957-0233/12/8/313.

[9] R. Kipping, R. Brito, E. Scheicher und U. Hampel. Developments for the application of the wire-mesh sensor in industries. International Journal of Multiphase Flow, 85:86-95, 2016. ISSN 0301-9322. https://doi.org/10.1016/j.ijmultiphaseflow.2016.05.017.

[10] H.-M. Prasser, A. Böttger und J. Zschau. A new electrodemesh tomograph for gas-liquid flows. Flow Measurement and Instrumentation, 9(2):111-119, 1998. ISSN 0955-5986. doi.org/10.1016/S0955-5986(98)00015-6.

[11] M. J. D. Silva, E. Schleicher und U. Hampel. Capacitance wire-mesh sensor for fast measurement of phase fraction distributions. Measurement Science and Technology, 18(7): 2245-2251, jun 2007. 10.1088/0957-0233/18/7/059.

[12] P. Wiedemann, A. Döß, E. Schleicher und U. Hampel. Fuzzy flow pattern identification in horizontal air-water two-phase flow based on wire-mesh sensor data. International Journal of Multiphase Flow, 117:153-162, 2019. ISSN 0301-9322. https://doi.org/10.1016/j.ijmultiphaseflow.2019.05.004.

[13] P. Wiedemann, F. d. A. Dias, E. Schleicher und U. Hampel. Temperature compensation for conductivity-based phase fraction measurements with wire-mesh sensors in gas-liquid flows of dilute aqueous solutions. Sensors, 20(24), 2020. ISSN 1424-8220. 10.3390/s20247114.

[14] H. Zhang, Y. Xiao und H. Gu. Numerical investigations of the accuracy of conductivity wire-mesh sensors. Nuclear Engineering and Design, 345:148-156, 2019. ISSN 0029-5493. doi.org/10.1016/j.nucengdes.2019.02.014. 